\title{
Diffusion of Linear Polymers within Microcapsules
}

\author{
Kristin E. Price; Steven J. Broadwater; Andrew R. Bogdan; Ivan Keresztes;
}

Jeremy L. Steinbacher; D. Tyler McQuade

\section{Table of Contents}

\begin{tabular}{|l|l|}
\hline Contents & Page \\
\hline NMR Diffusion Experiment Conditions & S1 \\
\hline Determination of Diffusion Constants & S2 \\
\hline Relaxation Times for Relevant Samples & S2 \\
\hline Diffusion Data for Each Sample & S3-S6 \\
\hline
\end{tabular}

\section{NMR Diffusion Experiment Conditions:}

Sample
Triphenylphosphine
TPP-PS
TPP-PS
TPP-PS Cap
Tris-(o-tolyl)phosphine
OTP-PS
OTP-PS
OTP-PS
OTP-PS Cap

$\begin{array}{cc}\text { Concentration } & \text { Temp } \\ 27 \mathrm{mg} / \mathrm{mL} & 20^{\circ} \mathrm{C} \\ 25.6 \mathrm{mg} / \mathrm{mL} & 20^{\circ} \mathrm{C} \\ 88 \mathrm{mg} / \mathrm{mL} & 20^{\circ} \mathrm{C} \\ \text { Packed tube } & 20^{\circ} \mathrm{C} \\ 29 \mathrm{mg} / \mathrm{mL} & 20^{\circ} \mathrm{C} \\ 26.4 \mathrm{mg} / \mathrm{mL} & 20^{\circ} \mathrm{C} \\ 39.6 \mathrm{mg} / \mathrm{mL} & 21^{\circ} \mathrm{C} \\ 77.6 \mathrm{mg} / \mathrm{mL} & 20^{\circ} \mathrm{C} \\ \text { Packed Tube } & 20^{\circ} \mathrm{C}\end{array}$

Sweep Width
$10000 \mathrm{~Hz}$
$10000 \mathrm{~Hz}$
$10000 \mathrm{~Hz}$
$10000 \mathrm{~Hz}$
$10000 \mathrm{~Hz}$
$10000 \mathrm{~Hz}$
$10000 \mathrm{~Hz}$
$10000 \mathrm{~Hz}$
$10000 \mathrm{~Hz}$

$\begin{array}{cc}\text { Pulse Width } & \text { Acq Time } \\ 10.7 & 0.7 \mathrm{~s} \\ 10.7 & 0.3 \mathrm{~s} \\ 10.7 & 0.3 \mathrm{~s} \\ 10.7 & 0.3 \mathrm{~s} \\ 10.75 & 0.7 \mathrm{~s} \\ 9.75 & 0.3 \mathrm{~s} \\ 9.75 & 0.3 \mathrm{~s} \\ 9.75 & 0.3 \mathrm{~s} \\ 9.75 & 0.3 \mathrm{~s}\end{array}$

$\begin{array}{lcccccccc}\text { Sample } & \begin{array}{c}\text { Recycle } \\ \text { Delay }\end{array} & \Delta & \delta & \tau & \text { Steps } & \text { nt } & \text { Minimum g } & \text { Maximum g } \\ \text { Triphenylphosphine } & 40 \mathrm{~s} & 0.5 \mathrm{~s} & 2 \mathrm{~ms} & 0.8 \mathrm{~ms} & 10 & 1 & 0.0099 \mathrm{~T} / \mathrm{m} & 0.3358 \mathrm{~T} / \mathrm{m} \\ \text { TPP-PS } & 14 \mathrm{~s} & 1 \mathrm{~s} & 5 \mathrm{~ms} & 0.8 \mathrm{~ms} & 10 & 64 & 0.002 \mathrm{~T} / \mathrm{m} & 0.553 \mathrm{~T} / \mathrm{m} \\ \text { TPP-PS } & 14 \mathrm{~s} & 4 \mathrm{~s} & 5 \mathrm{~ms} & 0.8 \mathrm{~ms} & 10 & 64 & 0.002 \mathrm{~T} / \mathrm{m} & 0.553 \mathrm{~T} / \mathrm{m} \\ \text { TPP-PS Cap } & 14 \mathrm{~s} & 5 \mathrm{~s} & 5 \mathrm{~ms} & 0.8 \mathrm{~ms} & 8 & 180 & 0.002 \mathrm{~T} / \mathrm{m} & 0.553 \mathrm{~T} / \mathrm{m} \\ \text { Tris-(o-Tolyl)phosphine } & 20 \mathrm{~s} & 0.5 \mathrm{~s} & 2 \mathrm{~ms} & 0.8 \mathrm{~ms} & 10 & 6 & 0.0099 \mathrm{~T} / \mathrm{m} & 0.3358 \mathrm{~T} / \mathrm{m} \\ \text { OTP-PS } & 14 \mathrm{~s} & 1 \mathrm{~s} & 5 \mathrm{~ms} & 0.8 \mathrm{~ms} & 10 & 64 & 0.002 \mathrm{~T} / \mathrm{m} & 0.553 \mathrm{~T} / \mathrm{m} \\ \text { OTP-PS } & 14 \mathrm{~s} & 2 \mathrm{~s} & 5 \mathrm{~ms} & 0.8 \mathrm{~ms} & 10 & 64 & 0.002 \mathrm{~T} / \mathrm{m} & 0.553 \mathrm{~T} / \mathrm{m} \\ \text { OTP-PS } & 14 \mathrm{~s} & 4 \mathrm{~s} & 5 \mathrm{~ms} & 0.8 \mathrm{~ms} & 10 & 64 & 0.002 \mathrm{~T} / \mathrm{m} & 0.553 \mathrm{~T} / \mathrm{m} \\ \text { OTP-PS Cap } & 14 \mathrm{~s} & 5 \mathrm{~s} & 5 \mathrm{~ms} & 0.8 \mathrm{~ms} & 8 & 180 & 0.002 \mathrm{~T} / \mathrm{m} & 0.553 \mathrm{~T} / \mathrm{m}\end{array}$




\section{Determination of Diffusion Constants:}

$$
\ln \left(\frac{I}{I_{o}}\right)=-D^{*} g^{2} * \gamma^{2} * \delta^{2} *\left(\Delta+\frac{4}{3} \delta+\frac{3}{2} \tau\right) \Rightarrow \ln \left(\frac{I}{I_{o}}\right)=-D^{*} g^{2} * k \Rightarrow \ln \left(\frac{I}{I_{o}}\right)=-D^{*} Q
$$

Definition of Variables:

$g=$ gradient strength in Tesla/m

$\gamma=$ magnetogyric ratio

$\delta=$ gradient length (total duration of bipolar gradient pulse)

$\Delta=$ diffusion delay

$\tau=$ gradient recovery delay

\section{Relaxation Times for the Molecules under Investigation:}

$\begin{array}{lccc}\text { Sample } & \text { Concentration* } & \mathbf{T}_{\mathbf{1}}(\mathbf{s}) & \mathbf{T}_{\mathbf{2}}(\mathbf{s}) \\ \text { Triphenylphosphine } & 25.9 \mathrm{mg} / \mathrm{mL} & 17.4 & 6.0 \\ \text { TPP-PS } & 88 \mathrm{mg} / \mathrm{mL} & 3.96 & 0.56 \\ \text { TPP-PS Cap } & \text { Packed tube } & 4.06 & 0.18 \\ \text { Tris-(o-tolyl)phosphine } & 31.5 \mathrm{mg} / \mathrm{mL} & 9.07 & 4.9 \\ \text { OTP-PS } & 40 \mathrm{mg} / \mathrm{mL} & 5.42 & 0.70 \\ \text { OTP-PS Cap } & \text { Packed tube } & 6.32 & 0.65 \\ \text { TPP-PS Bead } & \text { Packed tube } & 5.33 & <0.02\end{array}$

*The concentrations are exact for the $\mathrm{T}_{2}$ measurements. The polymer $\mathrm{T}_{1}$ values were collected at lower concentrations. 
NMR Diffusion Data: Error reflects deviation in diffusion rates, does not take $\mathrm{r}^{2}$ into account.

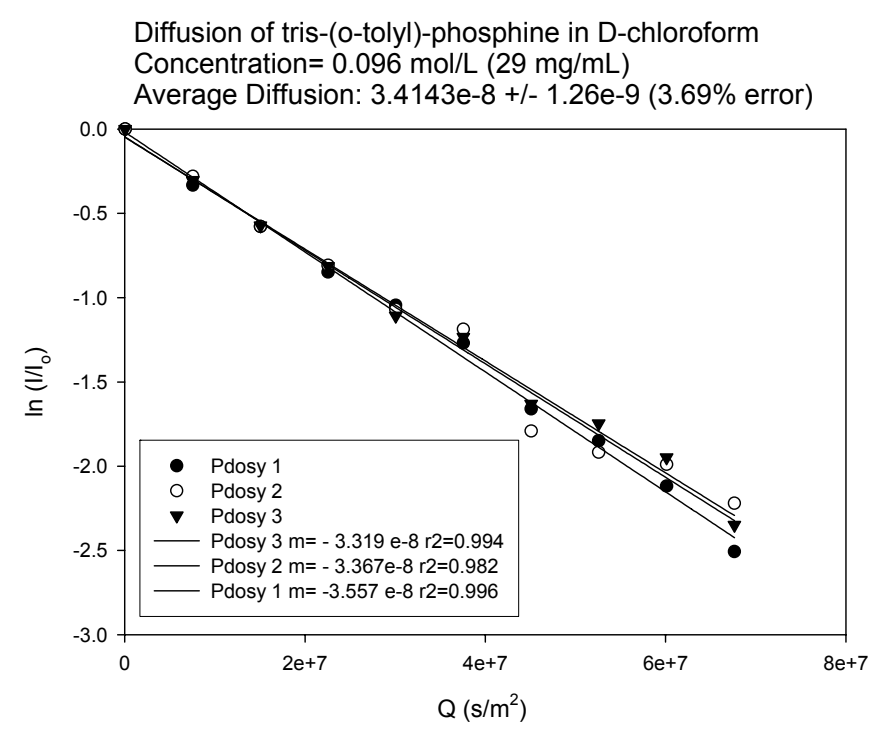

Diffusion of o-tolyl-phosphine Modified Polystyrene in Chloroform Concentration $=26.4 \mathrm{mg} / \mathrm{mL}$

Average Diffusion: $7.0367 \mathrm{e}-10+/-2.49 \mathrm{e}-11$ (3.54\% error)

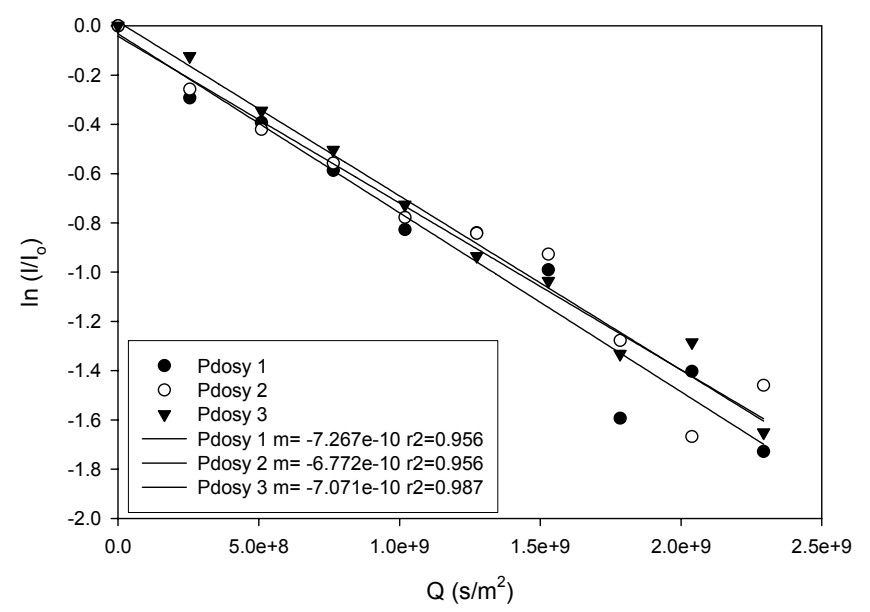




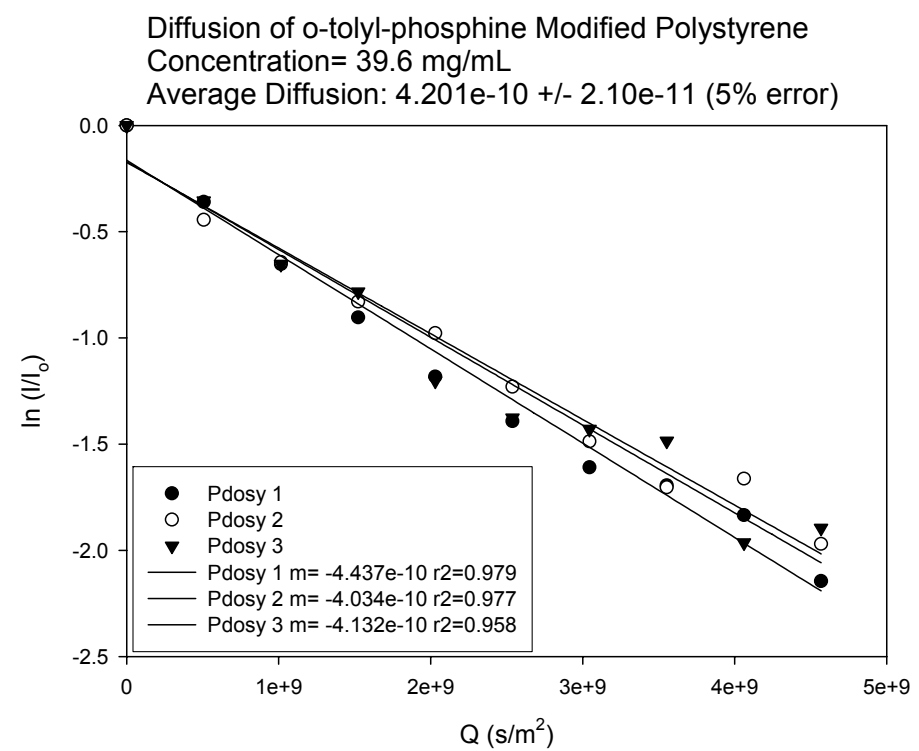

Diffusion of o-tolyl-phosphine Modified Polystyrene in Chloroform Concentration $=77.6 \mathrm{mg} / \mathrm{mL}$

Average Diffusion: $1.7097 \mathrm{e}-10+/-1.22 \mathrm{e}-11$ (7.14\%)

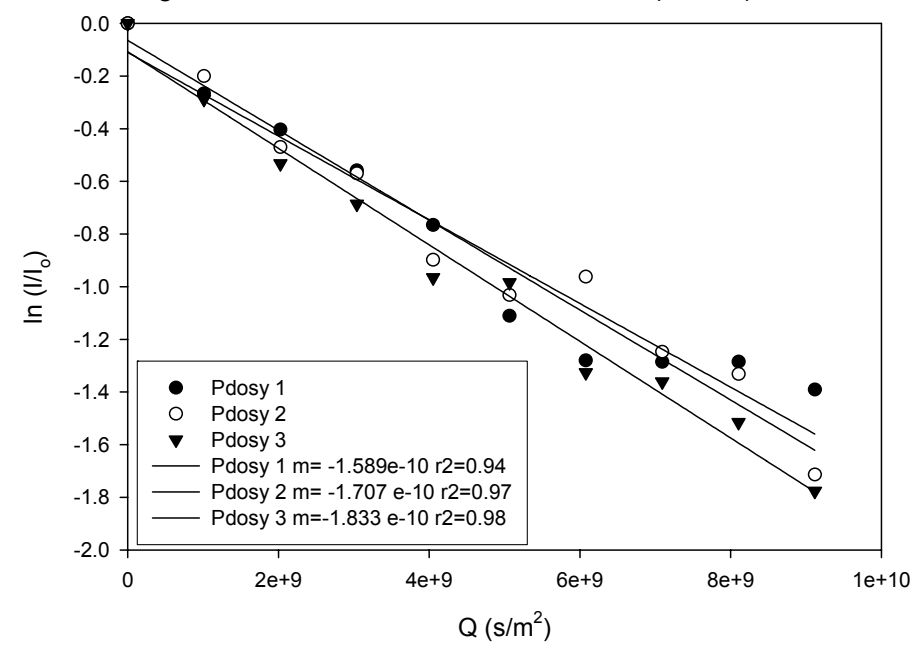

Diffusion of Triphenylphosphine in D-Chloroform

Concentration $=0.102 \mathrm{~mol} / \mathrm{L}(27 \mathrm{mg} / \mathrm{mL})$

Average Diffusion: $3.470 \mathrm{e}-8+/-1.45 \mathrm{e}-9$ (4.18\% error)

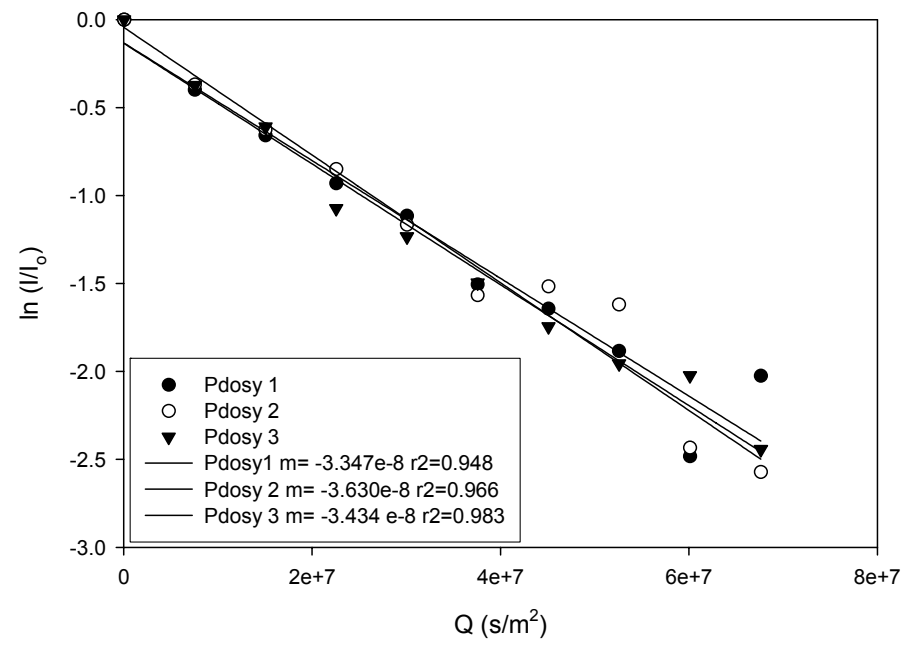


Diffusion of Triphenylphosphine Modified Polystyrene in Chloroform Concentration $=25.6 \mathrm{mg} / \mathrm{mL}$

Average Diffusion: $6.832 \mathrm{e}-10$ +/- 3.99 e-11 (5.85\% error)

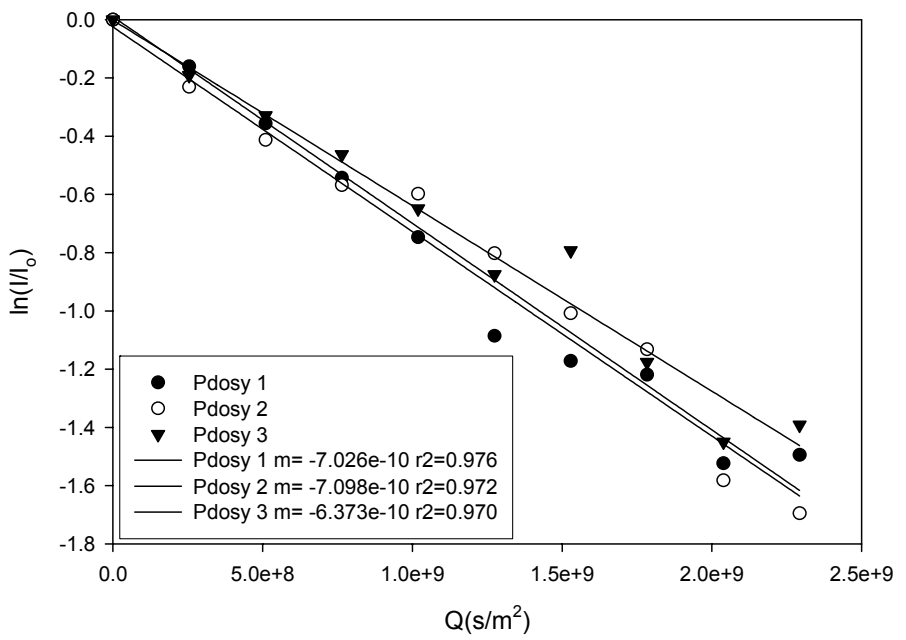

Encapsulated o-Tolyl Phosphine Modified Polystyrene Average Diffusion: 8.312e-11 +/- 1.85e-12 (2.23\% error)

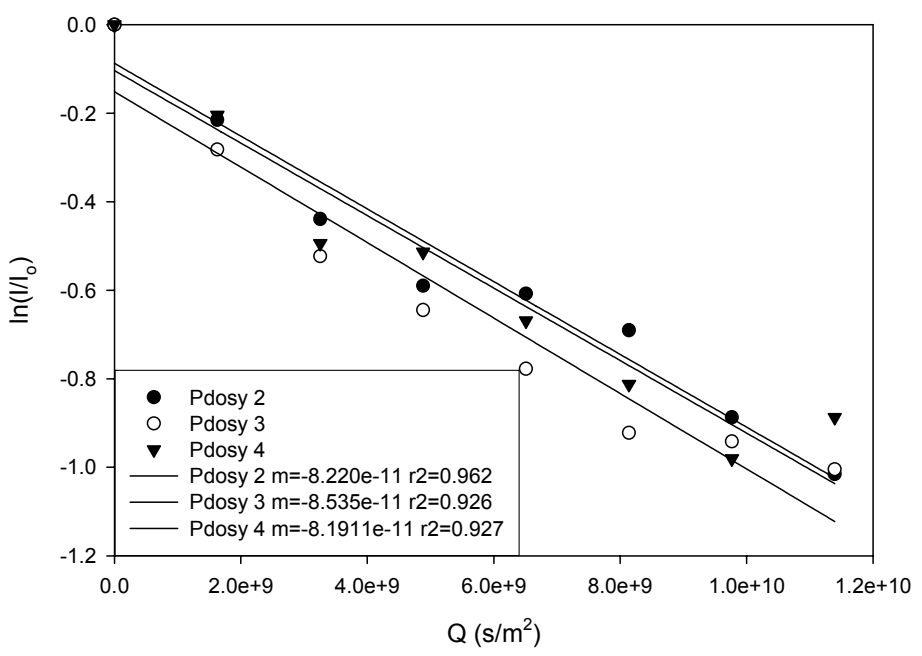

Encapsulate Triphenylphosphine Modified Polystyrene Average Diffusion $=5.92$ e- $11+/-1.21$ e- 11 (20\%error) (Over multiple NMR samples and capsule batches10 DOSY runs are averaged- $5.817 \mathrm{e}-11$ )

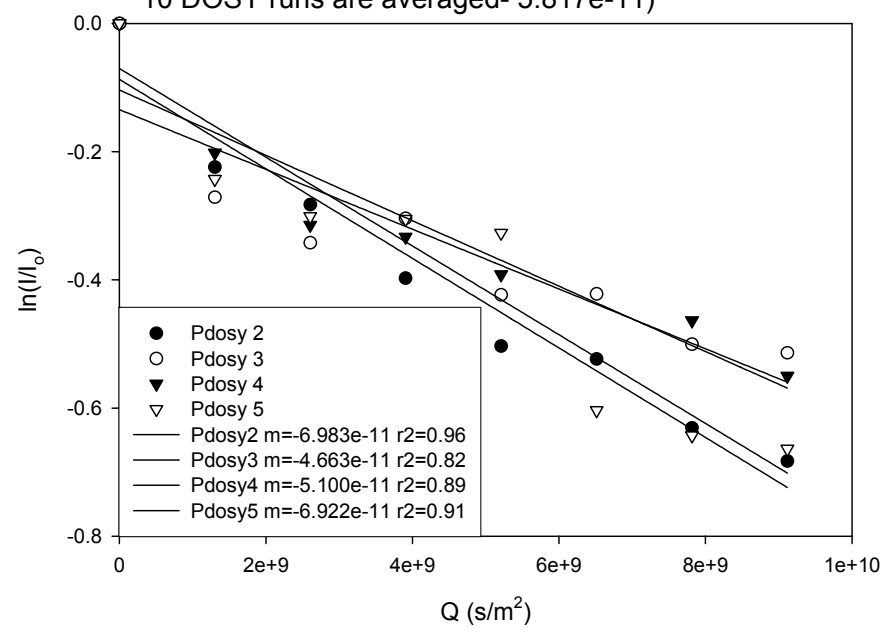




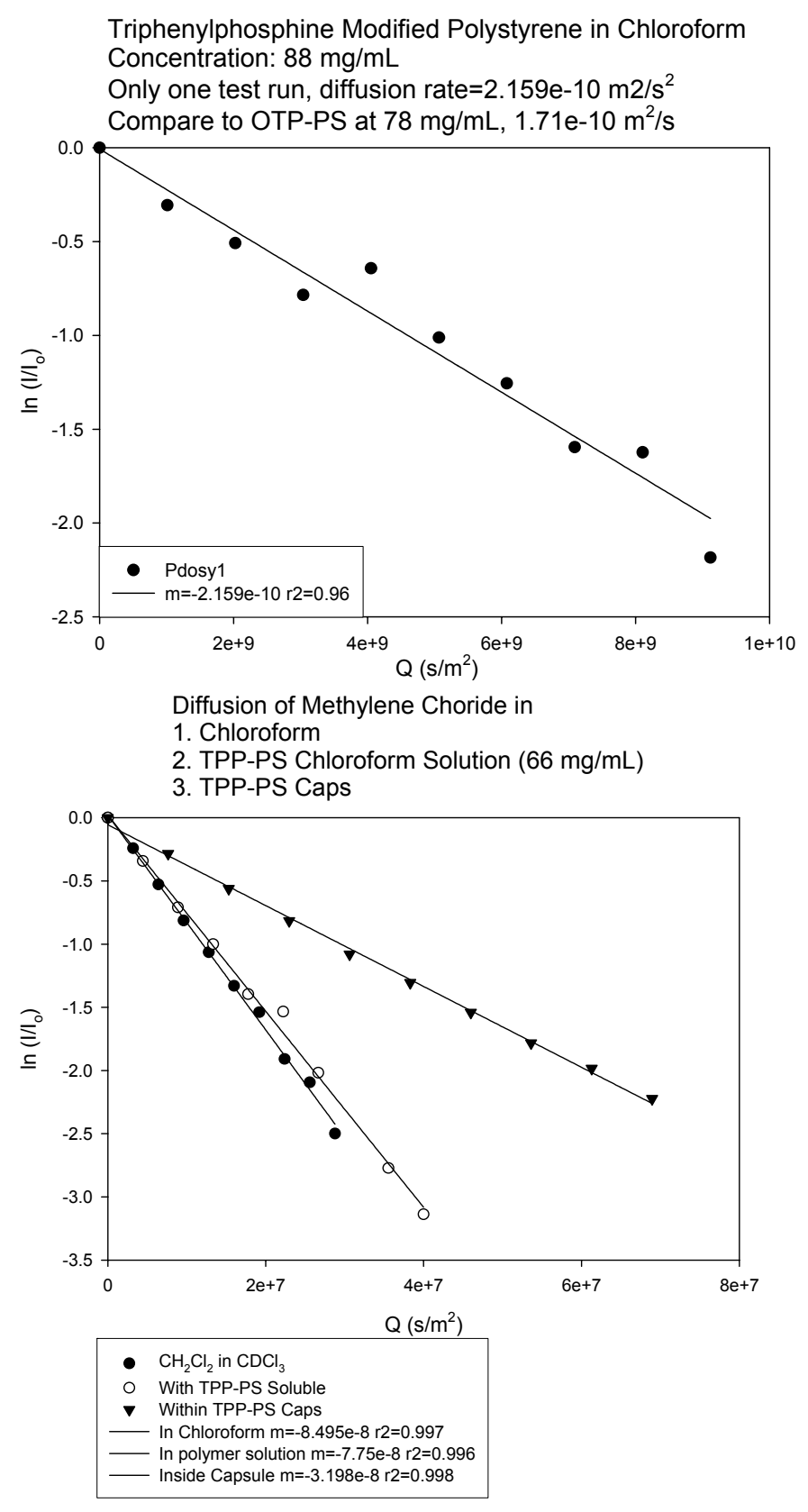

